\title{
LXIV. Laterally loaded struts and tie-rods
}

\section{Arthur Morley M.Sc.}

To cite this article: Arthur Morley M.Sc. (1908) LXIV. Laterally loaded struts and tie-rods, Philosophical Magazine Series 6, 15:90, 711-720, DOI: 10.1080/14786440809463811

To link to this article: http://dx.doi.org/10.1080/14786440809463811

册 Published online: 16 Apr 2009.

Submit your article to this journal $\pi$

Џll Article views: 4

Q View related articles $₫$ 
disturbing the relation of temperature and density at any point of the mass.

Equations (59) and (60) show that in spherical gaseous nebulas for whose gaseous stuff $\kappa=5$ the total internal energy, and the gravitational work, corresponding to each equilibrium distribution of gas, has the same value, whatever be the central temperature or total mass, provided temperature and density at each point within the mass are related to each other in accordance with the same value of the adiabatic constant in each case.

[To be continued.]

LXIV. Laterally loaded Struts and Tie-rods. By ARTHUR Morley, M.Sc., Professor of Mechanical Engineering at University College, Nottingham*.

THE frequent occurrence of laterally loaded struts and 1 ties in structures and machines makes the subject one of some importance. Every horizontal strut or tie-rod carries a lateral load in its own weight, every vertical stanchion which carries a horizontal wind load, every beam which is not horizontal, and the coupling-rods of locomotives loaded transversely by their own centrifugal force, are common examples. The increase in the maximum intensity of stress due to a small transverse load on a strut which has a considerable axjal thrust is very marked in long struts.

Prof. Perry $\dagger$ has given a method of finding the stresses in such cases, his solution embracing conditions of the most general kind.

Three methods are in common use for the determination of the maximum intensity of stress in such cases. In each of these the resultant intensity of stress is found by taking the algebraic sum of that due to the axial thrust or pull and the intensity of bending stress. The methods differ in the calculation of the bending stress only; the usual assumptions are made and the simple Bernoulli-Euler theory employed; but the calculation of the maximum bending moment differs in the three methods as follows:-

(a) The maximum bending moment is taken as that due to the transverse loads only. This will evidently be nearly correct in a very short stiff beam having a small thrust or pull.

(b) To the bending moment which the lateral loads would

* Communicated by the Author.

+ Phil. Mag. March 1892.

3 B 2 
alone produce is added that which would be caused by the axial load if the deflexions of the beam were due to the transverse loads only and were unaffected by the axial loads. This is the method usually given in the text-books for other than very short beams.

(c) The bending moment resulting from the axial loads is estimated by means of the deflexions calculated from the axial and lateral loads jointly, and is added to that resulting from the lateral loads. This is the method adopted in the paper mentioned above; but in the common case chosen for illustration an approximation is made in estimating the deflexion.

\section{Object of this Paper.}

The present paper is mainly concerned with the simple but most important cases of uniformly distributed and single concentrated loads and simple conditions of end support; its main object is to record the more exact solutions and to examine under what circumstances the simpler methods of calculation are approximately correct and to indicate the degree of approximation.

\section{Notation.}

The axis of $x$ is taken through the centres of area of the two ends of the bar and the origin is midway between these points. The length of the strut or tie-rod in all cases is taken as $l$. The axial force is $+\mathrm{P}$ a thrust in the case of a strut, and $-\mathrm{P}$ a pull in the case of a tie-rod. The radius of gyration of the area of cross section $A$ about a central axis perpendicular to the plane of bending is $k$, and the moment of inertia, $k^{2} \mathrm{~A}$, of the area $\mathrm{A}$ about the same axis is I. Only sections symmetrical about this axis are considered and the depth of section is taken as $d$. The average intensity of stress over the section is $p_{0}= \pm \frac{\mathrm{P}}{\mathrm{A}}$.

Since the curvature is always small within the limits of elasticity, it is taken as $\frac{d^{2} y}{d x^{2}}$, and the bending moment, reckoned negative when it tends to bend the bar concave towards its undeflected position, is equal to $\mathrm{EI} \frac{d^{2} y}{d x^{2}}$, where $\mathrm{E}$ is the modulus of direct elasticity. 
Case I.

Uniform straight strut with uniformly distributed load $w$ per unit length, and ends freely hinged.

$$
\begin{aligned}
& \mathrm{EI} \frac{d^{2} y}{d x^{2}}=-\frac{w}{2}\left(\frac{l^{2}}{4}-x^{2}\right)-\mathrm{P} y, \quad . . \\
& \frac{d^{2} y}{d x^{2}}+\frac{\mathrm{P}}{\mathrm{EI}} y=-\frac{w}{2 \mathrm{EI}}\left(\frac{l^{2}}{4}-x^{2}\right) .
\end{aligned}
$$

The conditions being $y=0$ for $x=\frac{l}{2}$ and $\frac{d y}{d x}=0$ for $a=0$, the solution is

$$
y=\frac{w}{2 \mathrm{P}} x^{2}-\frac{w l^{2}}{8 \mathrm{P}}-\frac{w \mathrm{EI}}{\mathrm{P}^{2}}\left(1-\sec _{\overline{2}}^{l} \sqrt{\frac{\overline{\mathrm{P}}}{\mathrm{EI}}} \cos \sqrt{\frac{\overline{\mathrm{P}}}{\mathrm{EI}}} x\right), .
$$

and at the origin

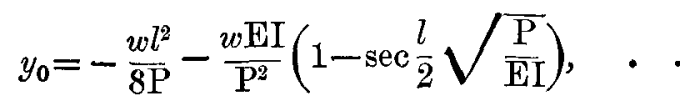

and the bending moment with sign reversed is

$$
\begin{aligned}
\mathrm{M}_{0} & =\mathrm{P} y_{0}+\frac{1}{8} w l^{2}=\frac{w \mathrm{EI}}{\mathrm{P}}\left(1-\sec _{\overline{2}}^{l} \sqrt{\overline{\mathrm{P}}}\right), . \\
\text { or } \quad \mathrm{M}_{0} & =\frac{w \mathrm{EI}}{\mathrm{P}}\left(\sec \frac{\pi}{2} \sqrt{\frac{\mathrm{P}}{\mathrm{P}}}-1\right), . . .
\end{aligned}
$$

where $\mathrm{P}_{e}$ is Euler's limiting value of $\mathrm{P}$ for the ideal strut, in this case $\frac{\pi^{2} \mathrm{EI}}{l^{2}}$, which value makes $\mathrm{M}$ and $y$ infinite.

The actual working value of $\frac{\mathrm{P}}{\mathrm{P}_{e}}$ will rarely reach as much as $\frac{1}{4}$ when there is no lateral load, and with a lateral load $\frac{1}{5}$ may be taken as an extreme value. As the resistance to bending under end thrust alone in a plane perpendicular to that of the lateral loads will often be less than that to bending under end thrust in the plane of the lateral loads, $\frac{P}{\mathrm{P}_{e}}$ will often be much less than $\frac{1}{5}$, as for example in a rectangular section the depth of which is considerably greater than the breadth. 
Applying the expansion

$$
\begin{gathered}
\sec \theta-1=\frac{\theta^{2}}{2 !}+\frac{5 \theta^{4}}{4 !}+\frac{61 \theta^{6}}{6 !}+\frac{1385 \theta^{8}}{8 !}+\ldots \text { \&c. to }(6), \\
\mathrm{M}_{0}=\frac{w l^{2}}{8}\left\{1+\frac{5 \pi^{2}}{48} \frac{\mathrm{P}}{\mathrm{P}_{e}}+\frac{61 \pi^{4}}{5760}\left(\frac{\mathrm{P}}{\mathrm{P}_{e}}\right)^{2}+\frac{277 \pi^{6}}{258048}\left(\frac{\mathrm{P}}{\mathrm{P}_{e}}\right)^{3}+\ldots \& c .\right\} .
\end{gathered}
$$

the coefficients of the various powers of $\frac{\mathrm{P}}{\mathrm{P}_{e}}$ differing but little from unity. This or (5) may also be written

$$
\mathrm{N}_{0}=\frac{w l^{2}}{8}+\frac{5}{384} \frac{w l^{4}}{\mathrm{EI}} \cdot \mathrm{P}\left\{1+\frac{61 \pi^{2}}{600} \cdot \frac{\mathrm{P}}{\mathrm{P}_{e}}+\frac{277 \pi^{4}}{26880}\left(\frac{\mathrm{P}}{\mathrm{P}_{e}}\right)^{2}+\ldots \& \mathrm{c} .\right\}
$$

The form (8) shows in a striking manner the relation of the approximate method (b) to the correct method of calculating the bending stress and the order of the error involved in the former; the first two terms of (8) represent the maximum bending moment as estimated by method $(b)$, and the coeffcients of the powers of $\frac{\mathrm{P}}{\mathrm{P}_{e}}$ are nearly unity; also the first term of (7) or (8) represents the maximum bending moment as estimated in the method $(a)$.

The equal-and opposite intensities of maximum bending stress. in.a symmetrical section are

$$
p_{b}= \pm \frac{\mathrm{M}_{0}}{\mathrm{Z}}= \pm \frac{w \mathrm{E} d}{2 p_{0} \mathrm{~A}}\left(\sec \frac{l}{2 k} \sqrt{\frac{p_{0}}{\mathrm{E}}-1}\right)
$$

where $\mathrm{Z}=\frac{2 \mathrm{I}}{d}$ is the modulus of section.

The maximum intensity of the compressive stress is

$$
f_{c}=p_{b}+p_{0}=\frac{w \mathrm{E} d}{2 p_{0} \mathrm{~A}}\left(\sec \frac{l}{2 k} \sqrt{\frac{p_{0}}{\mathbf{E}}-1}\right)+p_{0}
$$

and the maximum intensity of the tensile stress is

$$
f_{t}=p_{b}-p_{0}=\frac{w \mathrm{E} d}{2 p_{0} \mathrm{~A}}\left(\sec \frac{l}{2 \dot{k}} \sqrt{\frac{p_{0}}{\mathrm{E}}}-1\right)-p_{0}
$$

which may be positive or negative.

If $\frac{\mathrm{P}}{\mathrm{P}_{e}}=\frac{1}{5}$ the error involved in calculating the bending stress intensities by method (a) is 20 per cent., and by method (b) 4 per cent.; practically the same proportions it may be noticed in either case that the first neglected term in the 
series bears to the first term. The errors involved in calculating $f_{c}$ the maximum intensity of stress due to the axial and lateral forces combined will be less than these amounts, the error depending on the amount of the lateral compared with the axial load, and the shape of cross section. Similarly the error in calculating $f_{t}$ will be greater than the above amounts.

Prof. Perry's approximation mentioned above consists in substituting $\frac{w l^{2}}{\gamma} \cos \frac{x}{l} \pi$ for $\frac{w}{2}\left(\frac{l^{2}}{4}-x^{2}\right)$ in (1), which is equivalent to substituting a smaller but not a uniformly distributed load $\frac{\pi}{4} w l$ for the actual lateral load $w l$. This gives

$$
y_{0}=\frac{w l^{2}}{8\left(\mathrm{P}_{e}-\mathrm{P}\right)} \text { and } \mathrm{M}_{0}=\frac{1}{8} w l^{2} \cdot \frac{\mathrm{P}_{e}}{\mathrm{P}_{e}-\mathrm{P}},
$$

the deflexion being rather below the true value for all values of $P$. The most serious error arising from this approximation is that in calculating the bending stress for high ratios of $-\frac{\mathrm{P}}{\mathrm{P}_{e}}$ assuming the limits of elasticity not to be exceeded. For $\frac{\mathrm{P}}{\mathrm{P}_{e}}=0.9$ the error is $3 \frac{1}{2}$ per cent. on the deflexion and somewhat less on the bending stress; that on the maximum tensile stress $f_{t}$ is proportionally more than that on the bending stress.

Case II.

Uniform straight strut with central lateral load W and ends freely hinged.

The equation in this case is

$$
\frac{d^{2} y}{d x^{2}}+\frac{\mathrm{P}}{\mathrm{EI}} \cdot y=-\frac{\mathrm{W}}{2 \mathrm{EI}}\left(\frac{l}{2}-x\right), \quad . \quad . .
$$

and the conditions being as before,

$$
y_{0}=\frac{\mathrm{W}}{2 \mathrm{P}} \sqrt{\frac{\overline{\mathrm{EI}}}{\mathrm{P}}} \tan \frac{l}{2} \sqrt{\frac{\mathrm{P}}{\mathrm{EI}}}-\frac{\mathrm{W} l}{4 \mathrm{P}}, \ldots
$$

and at the centre

$$
-\mathrm{M}_{0}=\frac{\mathrm{W}}{2} \sqrt{\overline{\mathrm{EI}}} \tan \frac{l}{2} \sqrt{\overline{\mathrm{P}}}, \ldots .
$$

$\mathrm{M}_{0}$ and $y_{0}$ being evidently infinite for $\mathrm{P}=\frac{\pi^{2} \mathrm{EI}}{l^{2}}=\mathrm{P}_{e}$. 
Using the expansion

$$
\begin{aligned}
\tan \theta & =\theta+\frac{1}{3} \theta^{3}+\frac{2}{15} \theta^{5}+\frac{17}{315} \theta^{7}+\ldots \\
\text { or }-\mathrm{M}_{0} & =\frac{\mathrm{W} l}{4}\left\{1+\frac{\pi^{2}}{12} \frac{\mathrm{P}}{\mathrm{P}}+\frac{\pi^{4}}{120}\left(\frac{\mathrm{P}}{\mathrm{P}_{e}}\right)^{2}+\frac{17 \pi^{6}}{20160}\left(\frac{\mathrm{P}}{\mathrm{P}_{e}}\right)^{3}+\ldots \& c .\right\}, \\
-\mathrm{M}_{0} & =\frac{\mathrm{W} l}{4}+\frac{\mathrm{W} l^{3}}{48 \mathrm{EI}} \cdot \mathrm{P}\left\{1+\frac{\pi^{2}}{10} \frac{\mathrm{P}}{\mathrm{P}_{e}}+\frac{17 \pi^{2}}{1680}\left(\frac{\mathrm{P}}{\mathrm{P}_{e}}\right)^{2}+\ldots \& \mathrm{cc}\right\}, .
\end{aligned}
$$

which very clearly shows the relation of the calculation by the method $(b)$ to the true bending moment. For $\frac{\mathrm{P}}{\mathrm{P}_{e}}=\frac{1}{5}$
the error is under 4 per cent.

Case III.

Uniform straight strut with uniformly distributed load $w$ per unit length and ends fixed in the direction of the axis of $x$.

The equation is

$$
\frac{d^{2} y}{d x^{2}}+\frac{\mathrm{P}}{\mathrm{EI}} \cdot y=-\frac{w}{2 \mathrm{EI}}\left(\frac{l^{2}}{4}-x^{2}\right)+\frac{\mathrm{M}_{1}}{\overline{\mathrm{EI}}}, \cdot .
$$

where $M_{1}$ is the bending moment at the ends. The conditions being $\frac{d y}{d x}=0$ for $x=0, y=0$ for $x=\frac{l}{2}$, and $\frac{d y}{d x}=0$ for $x=\frac{l}{2}$ the solution

$$
y=\frac{w}{2 \mathrm{P}}\left\{x^{2}-\frac{l^{2}}{4}+l \sqrt{\frac{\overline{\mathrm{EI}}}{\overline{\mathrm{P}}}} \operatorname{cosec} \frac{l}{2} \sqrt{\frac{\mathrm{P}}{\overline{\mathrm{EI}}}}\left(\cos \sqrt{\frac{\mathrm{P}}{\mathrm{EI}}} x-\cos \frac{l}{2} \sqrt{\frac{\mathrm{P}}{\mathrm{EI}}}\right)\right\}
$$

gives

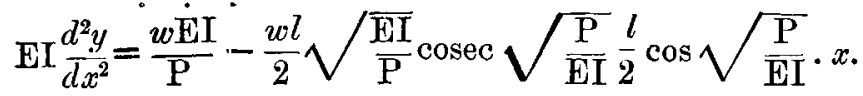

The points of inflexion are at a distance from the centro given by

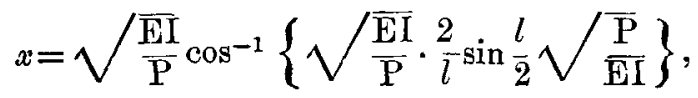

which varies from $\frac{l}{2 \sqrt{3}}$ when $\mathrm{P}=0$ to $\frac{l}{4}$ when $\mathrm{P}=\mathrm{P}_{e}=\frac{4 \pi^{2} \mathrm{EI}}{l^{2}}$.

Euler's limiting value for the ideal strut fixed at both ends. 
At the origin

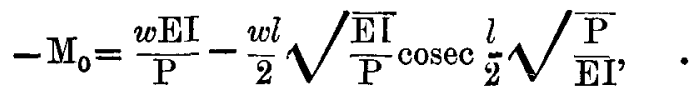

and the bending moment of greatest magnitude is at the fixed ends where

$$
\mathrm{M}_{1}=\frac{w \mathrm{EI}}{\mathrm{P}}-\frac{w l}{2} \sqrt{\frac{\mathrm{EI}}{\mathrm{P}}} \cot \frac{l}{2} \sqrt{\frac{\mathrm{P}}{\mathrm{EI}}} . .
$$

If the forms (16) and (17) are expanded they show a similar relation to those in cases I. and II. between the exact method of calculating the bending moment and method (b) if deflexions above or below the points of inflexion are used. These points as mentioned above vary somewhat in position according to the value of $\mathrm{P}$ and method (b) becomes less simple than the exact method.

Case IV.

Uniform straight strut with central load W and ends firmly fixed in the direction of the axis of $x$.

The equation is

$$
\frac{d^{2} y}{d x^{2}}+\frac{\mathrm{P}}{\mathrm{EI}} y=-\frac{\mathrm{W}}{2 \mathrm{EI}}\left(\frac{l}{2}-x\right)+\frac{\mathrm{M}_{1}}{\mathrm{EI}} \quad .
$$

where $M_{1}$ is the bending moment at the fixed ends. The solution gives the bending moment with sign reversed,

$$
\mathrm{M}=\frac{\mathrm{W}}{2} \sqrt{\frac{\mathrm{EI}}{\mathrm{P}}}\left\{\tan \frac{l}{4} \sqrt{\frac{\overline{\mathrm{P}}}{\mathrm{EI}}} \cos \sqrt{\frac{\overline{\mathrm{P}}}{\mathrm{EI}}} x-\sin \sqrt{\frac{\mathrm{P}}{\mathrm{EI}}} \cdot x\right\},
$$

which vanishes for all values of $\mathrm{P}$ when $x=\frac{l}{4}$.

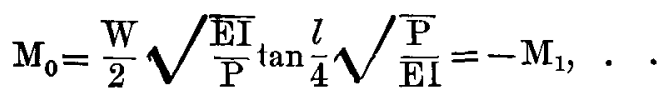

which becomes infinite for $\mathrm{P}=\frac{4 \pi^{2} \mathrm{EI}}{l^{2}}$.

And expanding (20),

$$
\mathrm{M}_{0}=-\mathrm{M}_{1}=\frac{\mathrm{Wl}}{8}+\frac{\mathrm{W} l^{3}}{384 \mathrm{EI}} \mathrm{P}\left\{1+\frac{4 \pi^{2}}{40}\left(\frac{\mathrm{P}}{\mathrm{P}}\right)+\frac{17 \pi^{4}}{1680}\left(\frac{\mathrm{P}}{\mathrm{P}_{e}}\right)^{2}+\ldots \& \mathrm{c} .\right\} \text {, }
$$

where $\mathrm{P}_{e}=\frac{4 \pi^{2} \mathrm{EI}}{l^{2}}$, Euler's limiting value of $\mathrm{P}$ for the ideal strut.

This again shows the relation of the method $(b)$ to the more exact method, the deflexions being reckoned above or below 
the points of inflexion which are at $x= \pm \frac{l}{4}$. The coefficients of powers of $\frac{\mathrm{P}}{\mathrm{P}_{e}}$ are nearly unity, and with any assigned ratio $\frac{\mathrm{P}}{\mathrm{P}_{e}}$ the error is easily estimated and with usual working ratios will be very small.

\section{Case V.}

Uniform straight tie-rod with uniformly distributed load $w$ per unit length ind ends freely hinged.

This is the same as case $I$. with the sign of $P$ reversed. The solution of the equation is

$y=-\frac{w}{2 \mathrm{P}} x^{2}+\frac{w l^{2}}{8 \mathrm{P}}-\frac{w \mathrm{EI}}{\mathrm{P}^{2}}\left(1-\operatorname{sech} \frac{l}{2} \sqrt{\frac{\overline{\mathrm{P}}}{\mathrm{EI}}} \cosh \sqrt{\left.\frac{\overline{\mathrm{P}}}{\mathrm{EI}} x\right)}\right.$,

and at the origin

$y_{0}=\frac{w l^{2}}{8 \mathrm{P}}+\frac{w \mathrm{EI}}{\mathrm{P}^{2}}\left(\operatorname{sech} \frac{l}{2} \sqrt{\frac{\mathrm{P}}{\mathrm{EI}}}-1\right)$.

and at the origin the bending moment with sign reversed,

$$
\mathrm{M}_{0}=\frac{w l^{2}}{8}-\mathrm{P} y_{0}=\frac{w \mathrm{EI}}{\mathrm{P}}\left(1-\operatorname{sech} \frac{7}{2} \sqrt{\frac{\mathrm{P}}{\mathrm{EI}}}\right) \text {, or } \frac{w \mathrm{EI}}{\mathrm{P}}\left(1-\operatorname{sech} \frac{\pi}{2} \sqrt{\frac{\overline{\mathrm{P}}}{\mathrm{P}_{e}}}\right) \text {, }
$$

where $\mathrm{P}=\frac{\pi^{2} \mathrm{EI}}{l^{2}}$.

And expanding (24),

$$
\begin{aligned}
& \mathrm{M}_{0}=\frac{w l^{2}}{8}\left\{1-\frac{5 \pi^{2}}{48} \cdot \frac{\mathrm{P}}{\mathrm{P}_{e}}+\frac{61 \pi \pi^{4}}{5760}\left(\frac{\mathrm{P}}{\mathrm{P}_{e}}\right)^{2}-\ldots \& c .\right\}, \ldots . . . \\
& \text { or } \\
& \mathrm{M}_{0}=\frac{w l^{2}}{8}-\frac{5}{384} \frac{w l^{4}}{\mathrm{EI}} \mathrm{P}\left\{1-\frac{61 \pi^{2}}{600}\left(\frac{\mathrm{P}}{\mathrm{P}_{e}}\right)+\frac{277 \pi^{4}}{26880}\left(\frac{\mathrm{P}}{\mathrm{P}_{e}}\right)^{2}-\ldots \& c .\right\}
\end{aligned}
$$

The errors involved in the use of the method $(b)$ are here about the same as in case $\mathrm{I}$. when $\frac{\mathrm{P}}{\mathrm{P}_{e}}$ is small, a condition which would not be fulfilled in a tie-bar carrying a reasonable pull unless $l$ is small or the lateral load is great. For tie-bars of considerable length (say $l$ greater than $20 d$ for circular sections) $\frac{\mathbf{P}}{\mathbf{P}_{e}}$ is generally greater than unity, and method $(b)$ is no longer of any use when the lateral load is only the weight of the bar; but, on the other hand, the bending stresses are then small compared with the tension resulting from the axial pull $P$. 
Prof. Perry's approximation gives

$$
y_{0}=\frac{w l^{2}}{8\left(\mathrm{P}_{e}+\mathrm{P}\right)} \text { and } \mathrm{M}_{0}=\frac{w l^{2}}{8} \cdot \frac{\mathrm{P}_{e}}{\mathrm{P}_{e}+\mathrm{P}} .
$$

For $\mathrm{P}=0$ this underestimates the deflexion by $2 \frac{1}{2}$ per cent. but gives the correct bending moment; for other values of $P$ it overestimates the value of the bending moment by a proportion which increases as $\mathrm{P}$ is increased; $e$. g., if $\mathrm{P}=\mathrm{P}_{e}$ the error is 2.6 per cent., and if $\frac{P}{P_{e}}=9$, a very possible value in a tie-rod of circular section, the error is over 12 per cent. If $l$ is increased indefinitely $\mathrm{M}_{0}$ according to this approximation approaches the limit $\frac{\pi^{2}}{8} \frac{w \mathrm{EI}}{\mathrm{P}}$ instead of $\frac{w \mathrm{EI}}{\mathrm{P}}$, as in (24), a limit of error of 23 per cent. on the bending moment. But in cases where $l$ is great the stress arising from the bending moment is usually unimportant compared with the axial tension, $w$ being small.

The intensity of bending stress

$$
p_{b}= \pm \frac{\mathrm{M}_{0}}{\mathrm{Z}}= \pm \frac{w \mathrm{E} d}{2 p_{0} \mathrm{~A}}\left(1-\operatorname{sech} \frac{l}{2 k} \sqrt{\overline{p_{0}}}\right)
$$

The maximum intensity of tensile stress is

$$
f_{t}=p_{b}+p_{0}=\frac{w \mathrm{E} d}{2 p_{0} \mathrm{~A}}\left(1-\operatorname{sech} \frac{l}{2 k} \sqrt{\frac{p_{0}}{\mathrm{E}}}\right)+p_{0} ; \quad
$$

and the maximum intensity of compressive stress is

$$
f_{c}=p_{b}-p_{0}=\frac{w \mathrm{E} d}{2 p_{0} \mathrm{~A}}\left(1-\operatorname{sech} \frac{l}{2 k} \sqrt{\frac{p_{0}}{\mathrm{E}}}\right)-p_{0}, \quad .
$$

which when negative gives the minimum intensity of tensile stress.

The pull $p_{0}$ per unit area of cross section which with given load and dimensions makes $f_{t}$ a minimum may bə found from (28), and the value of $p_{0}$ which makes $f_{c}$ zero may be found from (29).

\section{Case VI.}

Uniform straight tie-rod with central load $W$ and ends freely hinged.

The equation is

$$
\frac{d^{2} y}{d x^{2}}-\frac{\mathrm{P}}{\mathrm{EI}} \cdot y=-\frac{W}{2 \mathrm{E} \mathrm{I}}\left(\frac{l}{2}-x\right) . . . .
$$


The conditions being as in cases I., II., and V.,

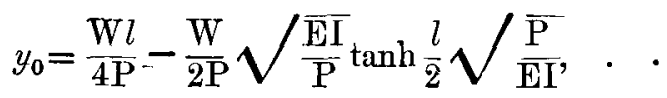

and the central bending moment

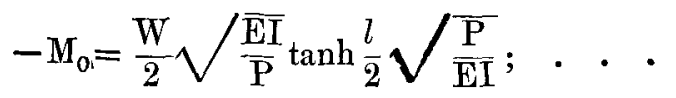

and expanding this

$$
\begin{aligned}
& -\mathrm{M}_{0}=\frac{\mathrm{W} l}{4}\left\{1-\frac{\pi^{2}}{12}\left(\frac{\mathrm{P}}{\mathrm{P}_{e}}\right)+\frac{\pi^{4}}{120}\left(\frac{\mathrm{P}}{\mathrm{P}_{e}}\right)^{2}-\frac{17 \pi^{6}}{20160}\left(\frac{\mathrm{P}}{\mathrm{P}_{e}}\right)^{3}+\ldots \& c .\right\}, \\
& \text { where } \mathrm{P}_{e}=\frac{\pi^{2} \mathrm{EI}}{l^{2}}
\end{aligned}
$$

or

$-\mathrm{M}_{0}=\frac{\mathrm{W} l}{4}-\frac{\mathrm{W} l^{3}}{48 \mathrm{EI}} \mathrm{P}\left\{1-\frac{\pi^{2}}{10}\left(\frac{\mathrm{P}}{\mathrm{P}_{e}}\right)+\frac{17 \pi^{4}}{1680}\left(\frac{\mathrm{P}}{\mathrm{P}_{e}}\right)^{2}-\ldots \& c_{.}\right\}$.

Here as in case V. for calculation of bending stresses method (b) is evidently a good approximation only when $\frac{\mathrm{P}}{\mathrm{P}_{e}}$ is small, $i$. e. when the bar is short or the lateral load great: for $\frac{\mathrm{P}}{\mathrm{P}_{a}}=\frac{1}{5}$ the error is 3 per cent.

Other cases with the same simple loadings as the above naturally suggest themselves, such for instance as those in which clamps fixing the ends at any assigned inclination to the axis of $x$ increase or decrease the flexure due to the lateral and axial loads, and those in which the longitudinal loads not passing through the centres of area of the ends of the strut or tie-rod may increase or decrease by their eccentricity the deflexion and bending moment resulting from the lateral loads.

LXV. The Changes in Velocity, in an Electric Field, of the $\alpha, \beta$ and Secondary Rays from Radioactive Substances. By A. S. Eve, M.A., Assistant Professor of Mathematics, and Lecturer in Radioactivity, Mc Gill University, Montreal* *

TN a paper on Secondary Radiation communicated to this 1 Magazine in December 1904, it was stated by me that the secondary rays from substances, due to the $\beta$ and $\gamma$ rays of radium, were homogeneous in character, and on that

* Communicated by the Author. 